REVISTA DEL INSTITUTO DE CIENCIAS JURÍdICAS
DE PUEBLA, MÉXICO, ISSN: 1870-2147. AÑO VIII NO. 34, JULIO-DICIEMBRE DE 2014 , PP. 21-41

\title{
La igualdad de género y el acceso a la justicia de las mujeres víctimas de violencia en la región dentro del sistema interamericano de protección de los derechos humanos*
}

\author{
Gender equality and access to justice \\ for women who are victims of violence \\ in the region, within the Inter-American \\ system for the protection of human rights
}

\author{
Patricia A. Taus**
}

\section{RESUMEN}

Desde un contexto regional y a partir de la vigencia del derecho internacional de los derechos humanos, el derecho inalienable a la igualdad es estudiado desde una perspectiva de género con el propósito de visualizar el ejercicio de jure y de facto del mismo. Se analizan los logros alcanzados en los últimos veinte años por el sistema interamericano de protección de los derechos humanos, a fin de dar solución al problema de la creciente, diversificada y multifacética violencia de género que impide la practica -real y no meramente formal-de la igualdad y la no discriminación.

PALABRAS CLAVE: Derechos humanos, igualdad, género, violencia, justicia, OEA.

\begin{abstract}
From a regional context and from the state of being in force of the International Law of Human Rights, the inalienable right to equality is studied from a gender perspective in order to view its legal and empirical exercise. It analyzes the achievements, in the last twenty years, by the Inter-American System for the Protection of Human Rights in order to solve the problem of growing, diversified and multifaceted gender violence that prevents the practice -real and not merely formal- of equality and non-discrimination.
\end{abstract}

KEY WORDS: Human rights, equality, gender, violence, justice, OAS.

\footnotetext{
* Recibido: 2 de mayo de 2014. Aceptado: 17 de junio de 2014.

** Colaboradora del Grupo Criminología y Justicia, México (drataus@yahoo.com.ar).
} 
A partir de la ratificación por parte de los Estados miembros de la Organización de Estados Americanos (en adelante, OEA) de los diversos tratados sobre derechos humanos con la consecuente vigencia regional del derecho internacional de los derechos humanos, pretendo analizar la igualdad desde una perspectiva de género a fin de comprobar los logros y adeudos presentes, tendentes a su ejercicio formal y real.

En principio, considero oportuno recordar que la igualdad de género, con la consecuente carencia de discriminación, es un derecho humano universal e inalienable previsto y tutelado en las diversas convenciones e instrumentos internacionales y regionales.

Pese a ello, la violencia de género se presenta como la vulneración más extendida de los derechos humanos, al representar una de las manifestaciones más extremas de la desigualdad y la discriminación. Si bien esta violación de los derechos humanos afecta a hombres, mujeres, niños y niñas, las segundas representan a los sujetos pasivos más numerosos a nivel mundial. En este sentido, lo que diferencia a este tipo de violencia de otras formas de agresión y coerción común es que el factor de riesgo o de vulnerabilidad está dado, principalmente, por el solo hecho de ser mujer, es decir, "[...] dirigida a personas o grupos de personas con base en su género". ' Esta violencia impacta de manera negativa en la identidad y bienestar social, físico y psicológico de la persona agredida.

Es dable destacar que diversas formas de violencia de género -si bien no todas- se consideran ilegales y actos criminales en las políticas y leyes nacionales de gran parte de los países del mundo. Así, el delito de violencia de género puede incluir "asaltos o violaciones sexuales, prostitución forzada, explotación laboral, el aborto selectivo en función del sexo, violencia física y sexual contra prostitutas, infanticidio femenino, tráfico de personas, violaciones sexuales durante periodo de guerra, ataques homofóbicos hacia personas o grupos de homosexuales, lesbianas, bisexuales y transgéneros".2

A nivel regional, el derecho a la igualdad y no discriminación se encuentra consagrado - entre otras normas- en la Convención Americana sobre Derechos Humanos (Pacto de San José de Costa Rica, de 1969), que estipula, principalmente, los derechos civiles y políticos, y establece dos mecanismos para la supervisión de los Estados en el respeto de los mismos, a saber:

a) La Comisión Interamericana de Derechos Humanos (en adelante, CIDH), creada en 1960, que actúa como órgano de la OEA y tiene competencia para

\footnotetext{
1 UnHCR-ACnUR. "Alto Comisionado de las Naciones Unidas para los Refugiados". [En línea]. Mayo de 2003, capítulo I, p. 10. [Citado: 1. Mayo. 2014]. Disponible en http://www.acnur.es/PDF/3667_20120402132757.pdf.

2 Engle Merry, Sally. "Violencia de género", en Forsythe, David P. Enciclopedia de Derechos Humanos (en inglés), Oxford University Press, 2009, vol. 1, p. 2428.
} 
controlar el cumplimiento de la Declaración Americana de los Derechos y Deberes del Hombre por parte de la totalidad de los Estados miembros de la OEA, y

b) La Corte Interamericana de Derechos Humanos (en adelante, Corte 1DH), establecida en 1978 cuando la Convención entró en vigencia. Al igual que la Comisión, tiene la facultad de examinar demandas individuales. Sin embargo, sólo puede tratar casos contenciosos una vez que los procedimientos seguidos ante la Comisión han finalizado y únicamente si la $\mathrm{CIDH}$ o un Estado le presenta el caso. Asimismo, emite opiniones consultivas a solicitud de los Estados o de los órganos de la oEA pertinentes. Las mismas pueden versar sobre la interpretación de la Convención u otros tratados sobre derechos humanos, o con respecto a la compatibilidad de las leyes nacionales con las obligaciones relativas a derechos humanos internacionales de los Estados miembros de la OEA.

Merece ser puesto de resalto que, muy recientemente, tanto la CIDH como la Corte IDH han podido percibir las violaciones a los derechos humanos de las mujeres y actuar en consecuencia. La Comisión se ha pronunciado con respecto a la discriminación en el derecho civil, penal y laboral, manteniendo una posición progresista en procura de la prohibición de la discriminación en todas las esferas. A modo de síntesis se puede mencionar:

- En el Informe Anual (1994) reconoció la falta de igualdad entre los hombres y las mujeres en el goce de los derechos humanos. ${ }^{3}$ A consecuencia de ello, designó a uno de sus miembros -Claudio Grossman- como Relator Especial sobre la Mujer y la Comisión empezó a incluir en los informes por país una sección relativa a los derechos humanos de la mujer.

- En el primer Informe por país sobre Haití (1995) analizó en detalle las consecuencias físicas, psicológicas y sociales de la violencia sexual contra la mujer en una situación de conflicto armado, junto a la caracterización jurídica de este fenómeno. Sostuvo que "las violaciones sexuales constituyen no sólo un tratamiento inhumano que atenta contra la integridad física, psíquica y moral, bajo el artículo 5 de la Convención, sino además una forma de tortura según el artículo 5 (2) del citado instrumento".4

\footnotetext{
3 OEA-CIDH. Informe Anual 1994. [En linea]. Nota 37, p. 219. [Citado: 3. Mayo. 2014]. Disponible en: http://www.cidh. oas.org/Informe1994.

4 OEA-CIDH. "Informe sobre Haiti". [En linea]. 1995, notas 33 y 28, p. 43. [Citado: 4. Mayo. 2014]. Disponible en: http:// www.cidh.oas.org/cidh/informepais/haiti95sp.
} 
- En el lnforme sobre Ecuador (1997) examinó “la violencia doméstica, la violación y el acoso sexual”. Puso énfasis en "la falta de protección judicial y en la necesidad de dictar medidas de prevención y apoyo a las mujeres maltratadas". ${ }^{5}$

- En el Informe sobre Brasil (1997), además de estudiar la violencia física, "incluyó los crímenes de honor y la falta de protección judicial para las mujeres víctimas de violencia doméstica". ${ }^{6}$

- En el Informe sobre México (1998) agregó "el problema de la violación marital y la inserción forzada de elementos intrauterinos".

- En el Informe sobre Colombia (1999) se refirió a "la violencia contra la mujer en los conflictos armados internos" e introdujo los problemas que enfrentan las colombianas en el área de la salud reproductiva, mencionando las consecuencias de la alta mortalidad materna como resultado del aborto o de la inseminación artificial forzada. ${ }^{8}$

- En el Informe sobre Perú (2000) continuó refiriéndose a los problemas de la salud reproductiva dentro de la sección sobre violencia, debido a la "esterilización forzada de las mujeres indígenas". ${ }^{9}$

- El primer caso relacionado con violación que resolvió fue el de "Raquel Martín de Mejía contra Perú" (Informe 5/96, 1996), donde concluyó que “el reiterado abuso sexual al cual fue sometida a manos de las fuerzas armadas constituyó una infracción del artículo 5 de la Convención, por lo que se constituye en tortura”. Asimismo, existió violación al artículo 11 de la Convención debido a que la violación es un "atropello a su dignidad". Señaló además diversas normas del derecho internacional humanitario y estableció la responsabilidad del Estado por la violación llevada a cabo por sus agentes..$^{10}$ Desafortunadamente, la Comisión no envió este caso a la Corte.

- Posteriormente, asumió el caso de "María Elena Loayza Tamayo contra Perú” (1997). Esta mujer también había sido presuntamente violada por

\footnotetext{
${ }^{5}$ OEA-CIDH. "Informe sobre Ecuador". [En línea]. 1997, nota 34, p. 122. [Citado: 5. Mayo. 2014]. Disponible en: http:// www.cidh.oas.org/cidh/informepais/ecuador97sp.

${ }^{6}$ OEA-CIDH. "Informe sobre Brasil". [En línea]. 1997, nota 34, párrafos 20-27, pp. 138-140. [Citado: 6. Mayo. 2014]. Disponible en: http://www.oas.org/cidh/informepais/brasil97sp.

7 OEA-CIDH. "Informe sobre México". [En línea]. 1998, nota 34, párrafos 619-629, pp. 141-144. [Citado: 7. Mayo. 2014]. Disponible en: http://www.cidh.org/countryrep/mexico98sp/indice.htm.

8 OEA-CIDH. "Informe sobre Colombia". [En línea]. 1999, nota 34, párrafos 35-39 y 47-54, pp. 328, 329, 331 y 332.

[Citado: 8. Mayo. 2014]. Disponible en: http://www.cidh.org/countryrep/colom99sp/indice.htm.

${ }^{9}$ OEA-CIDH. "Informe sobre Perú". [En línea]. 2000, nota 34, párrafos 18-26, pp. 185-188. [Citado: 9. Mayo. 2014].

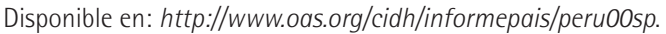

10 OEA-CIDH. "Raquel Martín de Mejía vs. Perú". [En línea]. Informe No. 5/96, Caso 10.970, 1 de marzo de 1996, capitulo III, apartado II-Análisis, punto a [Citado: 10. Mayo. 2014]. Disponible en: http://www.cidh.oas.org/annualrep/cap.III. peru 10.970.htm.
} 
agentes del Estado peruano en el momento en que se encontraba detenida por ser sospechosa de terrorismo, y acudió a la CIDH en 1993. A diferencia del anterior precedente, este caso fue enviado a la Corte con la conclusión, inter alia, de que "Perú habría infringido la disposición del artículo 5 de la Convención por las acciones de los funcionarios públicos que la habrían violado durante el transcurso de su detención”. Pese a ello, en 1999, la Corte no encontró pruebas de la comisión del delito de violación y no se explayó sobre el punto. ${ }^{11}$

Finalmente, y con el objeto de alcanzar una concepción objetiva de lo que acontece en los Estados miembros de la oEA respecto del acceso a la justicia de las mujeres víctimas de violencia, estimo pertinente referirme a lo reseñado en el trabajo realizado por la CIDH, Relatoría sobre Derechos de la Mujer, Doc. 68. ${ }^{12}$

La CIDH, en dicho Documento, reconoce los esfuerzos de los Estados regionales por adoptar un marco jurídico y político para abordar la violencia contra las mujeres, que incluye una gama de recursos e instancias judiciales de protección. No obstante, advierte una dicotomía entre su disponibilidad formal y su idoneidad para remediar dichos actos de violencia. En este sentido, ha constatado en la administración de justicia deficiencias en el cumplimiento de la obligación de la "debida diligencia", que conlleva ineficacia e impunidad ante el tratamiento de los casos de violencia contra las mujeres.

En varios países existe un patrón de impunidad sistemática en el procesamiento judicial y en las actuaciones relacionadas con la violencia contra las mujeres, que se comprueba con la carencia de investigación, sanción y reparación efectiva. La impunidad de estas violaciones de derechos perpetúa la aceptación social del fenómeno de la discriminación y violencia contra las mujeres como normal (y perteneciente a su vida privada); el sentimiento y la sensación de inseguridad femenino, así como una persistente desconfianza de ellas en el sistema de administración de justicia. Estas deficiencias se traducen en un número aún ínfimo de juicios orales y sentencias condenatorias que no corresponden a la prevalencia del problema. Desde esta óptica, la $\mathrm{CIDH}$, en el Informe de Fondo No. 54/01, Maria da Penha Maia Fernandes, aplicó la Convención de Belém do Pará y encontró que además de las violaciones en el caso individual, existía en Brasil un patrón de tolerancia estatal hacia casos de violencia doméstica, que se

\footnotetext{
11 oeA-Corte IDH, Caso "Loayza Tomayo vs. Perú". [En línea]. Resolución del 17 de noviembre de 1999, pp. 4-7. [Citado: 11. Mayo. 2014]. Disponible en: http://www.corteidh.or.cr/docs/casos/articulos/Seriec_60_esp.pdf.

12 Oea-CidH, Relatoría sobre Derechos de la Mujer. "Acceso a la justicia para mujeres víctimas de violencia en las Américas". [En línea]. Doc. 68, 20 de enero de 2007. [Citado: 12. Mayo. 2014]. Disponible en: http://www.cidh.oas.org/women/ Acceso07/cap2.htm.
} 
traducía en una ineficacia judicial para investigar y sancionar los casos. Enfatizó que "el deber del Estado de ejercer debida diligencia va más allá de su deber de sancionar y sentenciar, incluyendo también su deber de prevenir estas prácticas degradantes". ${ }^{13}$

En materia de prevención y protección, la ciDH ha verificado que las autoridades estatales, y en particular la policía, no cumplen plenamente con su deber de proteger a las mujeres víctimas de violencia contra actos inminentes. Se puede observar que la policía todavía no percibe el problema de la violencia contra las mujeres como prioritario dentro de la esfera de los delitos criminales. En este orden de ideas, una investigación auspiciada por el Fondo de Desarrollo de las Naciones Unidas para la Mujer (UNIFEM) y la CEPAL en los países del Caribe de habla inglesa, presentada durante la reunión de trabajo organizada por la Relatoría sobre Derechos de las Mujeres en Jamaica, revela "la utilización sumamente limitada de los poderes policiales para garantizar la implementación de la ley, en particular en casos de incesto y abuso de niñas". ${ }^{14}$ De la misma manera, destaca la persistencia y la aceptación por parte de agentes de la policía de normas patriarcales sobre la privacidad de los asuntos familiares.

La CIDH ha comprobado que la investigación se retrasa cuando las mujeres víctimas de violencia son reportadas como desaparecidas, y que las autoridades cometen dos clases de violaciones: 1) no proceden a buscar a la víctima con celeridad, y 2) la descalifican y culpabilizan por sus acciones y, por tanto, la señalan como no merecedora de acciones estatales para localizarla y protegerla. Este proceder disvalioso es particularmente grave cuando las víctimas son menores de edad.

En sendas investigaciones realizadas en cuatro países de la región -Chile, Guatemala, Honduras y Ecuador- se puso de relieve que las denuncias recibidas por el sistema son una pequeña parte de los hechos de violencia (física o sexual) en contra de las mujeres que se producen en cada país. Específicamente, la naturaleza de estos delitos, las percepciones sociales respecto de los mismos, la manera en que los operadores actúan frente a estos casos, las condiciones de recepción de las denuncias, los criterios de selección y persecución, la forma en que se practican los exámenes periciales, el no acompañamiento a las víctimas, las dificultades para acceder a las instituciones, la vulnerabilidad de las víctimas en el sistema, la poca respuesta que reciben, la duración de los casos,

\footnotetext{
${ }_{13}$ OEA-CIDH. Informe de Fondo "Maria da Penha Fernandes". [En línea]. No. 54/01, Caso 12.051, Brasil, 16 de abril de 2001, párrafo 56. [Citado: 16. Enero. 2007]. Disponible en: http://www.cidh.oas.org/informes.

14 oea-cidh y Fondo de Desarrollo de las Naciones Unidas para la Mujer. "Una mirada al acceso a la justicia en los países de habla inglesa", reunión de trabajo, Kingston, Jamaica, 29 y 30 de septiembre de 2005.
} 
etcétera, son algunas de las razones que explican la existencia de esta mayoritaria "cifra negra". ${ }^{15}$

Asimismo, en su Informe sobre la Situación de los Derechos Humanos de la Mujer en Ciudad Juárez, México (2003), la CIDH observó “parcialidades y sesgos de género presentes en las actuaciones de los fiscales e investigadores ante casos de violencia contra las mujeres, los cuales descalificaban a las víctimas durante el proceso de investigación”. De acuerdo con algunas declaraciones públicas de autoridades de alto rango, "las víctimas utilizaban minifaldas, salían de baile, eran fáciles o prostitutas". Hay informes acerca de que "la respuesta de las autoridades pertinentes frente a los familiares de las víctimas osciló entre indiferencia y hostilidad". ${ }^{16}$

Por ello, la CIDH destaca la necesidad de implementar campañas para sensibilizar al público en general sobre la gravedad de estos delitos y promover la interposición de denuncias. Sin embargo, considera que un importante obstáculo para que las mujeres víctimas de violencia accedan a instancias judiciales de protección es la vergüenza a la denuncia debido a la fuerte estigmatización que sufren.

Se observa que los sistemas de justicia no protegen de manera suficiente la dignidad y la privacidad de las víctimas dentro del proceso de investigación. Las mismas son revictimizadas por la existencia de una falta de sensibilidad ante su situación de víctimas, su sexo y la gravedad de los hechos alegados. A título ilustrativo, durante las reuniones de trabajo organizadas por la Relatoría sobre Derechos de las Mujeres se trató el caso de Nicaragua, donde "la autoridad que recibe la denuncia original, generalmente la policía, solicita que la víctima acuda al lnstituto de Medicina Legal y a un psicólogo para que pronuncien dictámenes, pero que por lo general éstos son descalificados por el agresor, quien por su parte ordena la realización de varios exámenes alternativos". ${ }^{17}$

En relación con los vacíos e irregularidades que afectan las investigaciones de casos de violencia contra las mujeres, la $\mathrm{CIDH}$ ha identificado la ausencia de pruebas físicas, científicas y psicológicas para establecer los hechos, lo cual se traduce en el estancamiento de los casos por falta de pruebas. Se mencionan como problemas persistentes el hecho de que los ministerios públicos se conforman con las siguientes pruebas: informes médicos de constatación de lesiones

\footnotetext{
${ }_{15}$ FARITO, SIMON C. y CASAS, LIDIA. "Evaluación de la reforma procesal penal desde una perspectiva de género", Centro de Estudios de Justicia de las Américas, México, noviembre de 2004, p. 8.

${ }^{16}$ OEA-CIDH. "Situación de los derechos humanos de la mujer en Ciudad Juárez, México: el derecho a no ser objeto de violencia y discriminación". [En línea]. oEA/Ser.L/V/II.117, 7 de marzo de 2003, Doc. 44, párrafo 4. [Citado: 17. Enero. 2007]. Disponible en: http://www.cidh.org/annualrep/2002sp/cap.vi.juarez.htm.

17 OeA-CidH e Instituto Interamericano de Derechos Humanos, "Una mirada al acceso a la justicia en los países de México y América central", reunión de trabajo, San José, Costa Rica, 11 y 12 de agosto de 2005.
} 
físicas y testimoniales, sin llevar a cabo "una eficiente recopilación de medios probatorios para la comprobación y reconstrucción fáctica del delito”. También manifiesta su preocupación sobre la "cadena de custodia" en casos de violencia y el énfasis exclusivo en preservar pruebas de carácter físico. Además, se observa la carencia de protocolos que describan la complejidad probatoria de estos casos.

En cuanto a las sanciones por el delito de violencia contra las mujeres, en algunos países la aplicación incorrecta, por parte de las fiscalías, del principio de oportunidad ignora la situación de vulnerabilidad y de desprotección en la que se encuentran las víctimas de violencia (miedo a represalias por parte del agresor y a la estigmatización pública). Esta situación y los peligros de ofrecer este amplio margen de discrecionalidad fueron mencionados durante las reuniones de trabajo organizadas por la Relatoría sobre Derechos de las Mujeres en discusiones sobre Paraguay ${ }^{18}$ y Guatemala. ${ }^{19} \mathrm{La}$ CIDH ha expresado su preocupación ante el hecho de que "una diversidad de órganos judiciales promueven principalmente el uso de la conciliación durante el proceso de investigación como método para resolver delitos de violencia contra las mujeres, sobre todo la intrafamiliar". ${ }^{20}$ Esto resulta peligroso debido a que en la conciliación se asume que las partes involucradas se encuentran en igualdad de condiciones de negociación, lo cual no sucede en el ámbito de la violencia intrafamiliar. "En varios países ha quedado claro que los acuerdos realizados en el marco de mediación aumentan el riesgo físico y emocional de las mujeres por la desigualdad en las relaciones de poder entre la víctima y el agresor". Los acuerdos generalmente "no son cumplidos por el agresor y éstos no abordan las causas y consecuencias de la violencia en sí. ${ }^{21}$

Por otra parte, la CIDH ha observado que la violencia, la discriminación y las dificultades para acceder a la justicia afectan en forma diferenciada a las mujeres. Desde la declaración oficial de Durban, se visibilizó que "[...] el racismo, la discriminación racial y la xenofobia se manifiestan de manera diferenciada en el caso de la mujer, haciendo que sus condiciones de vida se agraven, generando múltiples formas de violencia y limitando o negándoles el ejercicio de sus

\footnotetext{
18 oea-cidh y Relatoria sobre los Derechos de las Mujeres. "Una mirada al acceso a la justicia en los países del Cono Sur", reunión de trabajo, Senado de la Nación, Buenos Aires, Argentina, 12 y 13 de septiembre de 2005.

${ }^{19}$ Instituto Interamericano Derechos Humanos, "Una mirada al acceso a la justicia en los países de América Central y México", reunión de trabajo, San José, Costa Rica, 11-12 de agosto de 2005.

${ }^{20}$ OEA-CIDH. "Las mujeres frente a la violencia y la discriminación derivadas del conflicto armado en Colombia", OEA/ Ser.L//II. Doc. 67, 18 de octubre de 2006, párrafo 210.

${ }^{21}$ OEA-CIDH. "Violencia doméstica en Centroamérica", audiencia temática, 125 Perido Extraordinario de Sesiones, organizada por el Centro por la Justicia y el Derecho Internacional (CEIL) y organizaciones componentes de la Red Feminista Centroamericana contra la Violencia hacia las Mujeres-Las Dignas, Las Mélidas, ORMUSA y CemuJeres de El Salvador; cefemina de Costa Rica; el Centro de Derechos de Mujeres de Honduras; la Red de Mujeres contra la Violencia Nicaragua; la Red de Mujeres contra la Violencia de Panamá; la Red de la No Violencia contra las Mujeres de Guatemala, 19 de julio de 2006.
} 
derechos humanos". ${ }^{22}$ Lo dicho impacta directamente en las mujeres indígenas y afrodescendientes, debido a que están particularmente expuestas al menoscabo de sus derechos por causa del racismo. Los obstáculos que enfrentan para acceder a recursos judiciales idóneos y efectivos que remedien las violaciones sufridas pueden ser particularmente críticos porque padecen varias formas de discriminación combinadas: por ser mujeres, por su origen étnico o racial y/o por su condición socioeconómica.

Resulta pertinente recordar que en las Américas viven entre 45 y 50 millones de personas pertenecientes a más de 400 pueblos indigenas que conservan sus propios idiomas, visiones del mundo y formas de organización socio-política. Más del cincuenta por ciento son mujeres, la mayoría de ellas víctimas de doble discriminación, por ser mujeres y por ser indígenas. Las mujeres indígenas forman parte de sociedades culturalmente diferenciadas, constituidas a partir de su estrecha relación con sus territorios ancestrales y los recursos que allí se encuentran, no sólo por ser éstos su principal medio de subsistencia, sino además porque constituyen un elemento integrante de su cosmovisión.

La CIDH ha advertido sobre los obstáculos que enfrentan las mujeres indígenas para acceder a la justicia, generalmente relacionados con la exclusión social y discriminación étnica que han sufrido históricamente (escasez crónica de oportunidades y de acceso a servicios básicos de calidad, a los mercados laborales y de crédito, a condiciones físicas y de infraestructura adecuada, y al sistema de justicia). A ello se suma la dificultad que enfrentan por la inaccesibilidad geográfica de los territorios indígenas. Del testimonio de mujeres indígenas recopilado en Colombia, Guatemala, Honduras, Panamá y Paraguay, surge que deben realizar largas caminatas, de varios días inclusive, por tierra o por agua, para llegar a la ciudad más cercana a denunciar los hechos de violencia sufridos, lo que genera además dificultades de índole probatoria. Sin embargo, los problemas de acceso a la justicia no terminan con la llegada a la ciudad: ahí enfrentan otros de tipo económico, falta de información, y desconocimiento tanto de los contextos urbanos como del idioma utilizado en los tribunales.

Además, las mujeres indígenas se deben enfrentar al acceso a la justicia del Estado y al reconocimiento del derecho indígena. Aquí se presenta un dilema, ya que ambos sistemas pueden no ser compatibles con los derechos humanos internacionalmente reconocidos. $\mathrm{Al}$ respecto, la $\mathrm{CIDH}$ ha expresado la necesidad de que los Estados instauren y apliquen "un sistema judicial acorde con la diversidad cultural existente en los países, así como mecanismos que permitan

22 Conferencia Mundial contra el Racismo, la Discriminacion Racial, la Xenofobia y las Formas Conexas de Intolerancia, Ginebra, 15 y 16 de enero de 2001. 
reconocer y promover efectivamente el derecho indígena, conforme a sus normas tradicionales, tomando como parámetro las normas internacionales sobre derechos humanos". 23

En este orden de ideas resulta ejemplificador el Informe No. 53/01, Caso de las Hermanas González Pérez (2001), en donde la CIDH desarrolló el concepto de violación sexual como tortura y transgresión al derecho a la vida privada de las mujeres. Se refirió a los obstáculos específicos que las mujeres indígenas enfrentan al intentar acceder a instancias de protección judicial. La CIDH subrayó que el dolor y la humillación que sufrieron estas mujeres "se agravó por ser indígenas en razón del desconocimiento del idioma de sus agresores y de las demás autoridades intervinientes, y además por el repudio de su propia comunidad como consecuencia del delito de que fueron víctimas". ${ }^{24}$

Se citan como ejemplos preocupantes de la situación de violencia e impunidad que deben sobrellevar las mujeres indígenas, los casos de Colombia y Guatemala.

En Colombia, la situación de las mujeres indígenas "se ve agravada por el conflicto armado que utiliza como escenario sus territorios ancestrales, así como por los obstáculos que enfrentan para denunciar los hechos de violencia y discriminación que sufren". Dentro de este contexto, los pueblos indígenas han padecido "[...] masacres, asesinatos, en especial de sus líderes y autoridades tradicionales, secuestros y desplazamientos masivos de sus territorios ancestrales". Asimismo, la Relatoría ha recibido los testimonios de las denuncias sobre agresiones sexuales cometidas por los actores armados que participan en el conflicto, los cuales indican que la actitud discriminatoria del agresor agudiza el ya grave tipo de agresión: “[...] las patrullas de los diferentes grupos armados que ocupan los territorios indígenas raptan mujeres indígenas, las utilizan sexualmente en forma colectiva y luego las abandonan, amparándose en la impunidad de sus actos". ${ }^{25}$ Estos hechos, frecuentemente, no son denunciados porque los victimarios controlan los territorios en donde estos delitos ocurren, lo que provoca en las mujeres víctimas una obvia desconfianza en el sistema de justicia porque estiman que los delitos no serán investigados ni sus agresores sancionados. Unido a lo anterior se encuentra el desconocimiento de las mujeres sobre la ubicación de las instituciones donde deben entablar sus denuncias.

\footnotetext{
${ }^{23}$ OEA-CIDH. "Justicia e inclusión social en Guatemala: Ios desafios de la democracia en Guatemala", capítulo IV, párrafo 236.

${ }^{24}$ oEA-CIDH. "Informe de fondo México". [En línea]. No. 53/01, Caso 11.565 "Ana, Beatriz, y Cecilia González Pérez", 4 de abril de 2001, párrafo 95. [Citado: 27. Enero. 2007]. Disponible en: http://www.cidh.org/annualrep/.../fondo/ Mexico 11.565.htm.

${ }^{25}$ OEA-CIDH. "Las mujeres frente a la violencia y la discriminación derivadas del conflicto armado en Colombia", OEA/ Ser.L//II. Doc. 67, 18 de octubre de 2006, párrafos 123, 124 y 144.
} 
En Guatemala, las cifras de la exclusión social de los pueblos indígenas indican que el 46.4\% de las personas menores de edad padecen desnutrición crónica. Aquí, el problema no es sólo que un sector de la población no pueda ejercer su "libertad cultural", practicando su propia cultura, sino que además, “[...] históricamente no han gozado y no disfrutarán todavía del mismo rango de derechos sociales, económicos y políticos que el resto de sus compatriotas, por ser diferentes". En otras palabras, en el caso guatemalteco, "la diferencia va unida a la exclusión social en unos términos que no son reconocidos oficialmente ni aparecen en ninguna legislación". ${ }^{26}$

Por otra parte, según una reseña temática del Banco Mundial, de los 520 millones de personas que viven en América Latina y el Caribe (ALC), más de 150 millones son descendientes de africanos. ${ }^{27}$

La CIDH ha manifestado que en el caso particular de las mujeres afrocolombianas, su sexo ha implicado para ellas discriminación no sólo por ser afrocolombianas, sino también por ser mujeres, dentro y fuera de sus comunidades. ${ }^{28}$ "La discriminación es un dispositivo de control para mantener a los grupos marginados en una posición de subordinación”. Así, la violencia representa una de las peores consecuencias y estadios mayores de la discriminación. Por ello, se hace necesario "rechazar todas las formas de discriminación para avanzar en la erradicación de la discriminación por género". 29

Es dable destacar que en la recolección de datos oficiales se afecta a las personas afrodescendientes, diluyendo a esta población en distintos grupos o segmentos. Por ejemplo, en algunos países se les ha incluido dentro de la categoría grupo étnico, o bien como grupo indígena. Mientras que en Brasil las personas se autoidentifican por el "color o raza"; en Costa Rica lo hacen por la "cultura" a la cual pertenecen, y en Honduras por el "grupo poblacional" (garífuna y/o negro inglés). En Guatemala la autoidentificación se realiza con base en el grupo étnico al cual se considera perteneciente, y en Ecuador, si se

\footnotetext{
${ }_{26}$ Programa de Naciones Unidas para el Desarrollo. Informe Nacional de Desarrollo Humano 2005: "Diversidad étnicocultural: la ciudadanía en un Estado plural", Guatemala, 2005, p. 25. Véase, también, CEPAL-UNICEF. "Desnutrición infantil en América Latina y el Caribe. Desafíos", boletín 2, abril de 2006.

La desnutrición crónica, déficit de talla para la edad, refleja la acumulación de consecuencias de la falta de alimentación y nutrición adecuada durante los años más críticos del desarrollo de los niños - desde la etapa intrauterina hasta los 3 primeros años-. Sus efectos son, en gran medida, irreversibles y se relacionan estrechamente con la extrema pobreza.

27 Banco Mundial. Más allá de los promedios afrodescedientes en América Latina, Washington, D. C., febrero de 2006.

28 OEA-CIDH. "Las mujeres frente a la violencia y la discriminación derivadas del conflicto armado en Colombia", OEAl Ser.L//II. Doc. 67, 18 de octubre de 2006, párrafo 112.

29 UNESCO. "La protección de los derechos de la mujer en el sistema interamericano: un análisis del acceso a la justicia", presentación de Edna Santos Roland, coordinadora para Asuntos de Raza y Discriminación, Washington, D. C., 19 y 20 de abril de 2005.
} 
pregunta cómo "se considera" la persona, se tienen las siguientes opciones de respuesta: indigena, negro, afroecuatoriano, mestizo, mulato y otro. Además, en relación con la población afrodescendiente, los censos demográficos no investigan esta característica en todos los países de la región. Cuando se investiga la variable raza o color, la respuesta del entrevistado está condicionada a diversos factores, como por ejemplo los niveles de ingresos y educacionales, la conciencia de la negritud, la tendencia al "autoblanqueamiento", todo lo cual dificulta o imposibilita la comparabilidad de datos entre países. El problema de las cifras es vital. Sin datos confiables, sin indicadores y mediciones periódicas es imposible tomar decisiones políticas destinadas a enfrentar el problema de la discriminación. Las cifras, además, tienen un innegable componente político, pues para los afectados significa la visibilización de su situación, así como una forma de reconocimiento frente a los otros. ${ }^{30}$

Las mujeres afrodescendientes enfrentan un impacto diferenciado en comparación con los hombres, puesto que en ellas se entrecruza el componente de género con su pertenencia a una raza/etnia, lo que potencia su situación de segregación. La CIDH considera que un análisis del acceso a la justicia, para las mujeres afrodescendientes, implica considerar la gama de diferencias que subsisten al interior de esta población, las cuales están asociadas a su cosmovisión, a sus tradiciones y cultura, a su posición económica y geográfica, entre otras. De esta manera, las mujeres afrodescendientes ubicadas en zonas rurales marginadas, cohesionadas en grupos sociales pequeños, que aún mantienen sus idiomas, tradiciones y costumbres, y en ocasiones sus propios sistemas de justicia, deberán enfrentar problemas de accesibilidad geográfica, de falta de comunicación con los operadores judiciales en sus propios idiomas, de manejo del contexto, así como de falta de recursos económicos, similares a los que deben enfrentar las mujeres indígenas. Esta situación no dista de la que deben padecer las mujeres afrodescendientes ubicadas en zonas urbanas, donde las dificultades para acceder a remedios judiciales efectivos están asociadas con su situación de marginalidad económica y con el color de la piel. Consecuentemente, en aquellos espacios donde el factor económico y de marginación social ha sido superado, las dificultades generalmente están relacionadas con el color de la piel. ${ }^{31}$

Otro desafío, verificado por la $\mathrm{CIDH}$, que enfrentan las mujeres afrodescendientes es la violencia institucional perpetrada por autoridades judiciales que

\footnotetext{
30 Naciones Unidas-Cepal, División de Desarrollo Sostenible. Los pueblos indígenas y afrodescendientes ante el nuevo milenio, Santiago de Chile, abril de 2006, p. 25.

31 Banco Mundial-Grupo de Analisis para el Desarrollo (agrade). Más allá de los promedios afrodescedientes en América Latina. Pobreza, discriminación social e identidad. El caso de la población afrodescendiente en el Perú, Washington, D. c., febrero de 2006.
} 
no comprenden su cosmovisión, tradiciones y cultura. En este sentido, se hace necesario promover una cultura judicial que tolere la diferencia y la diversidad. ${ }^{32}$ Sumado a ello, como estos grupos se ven particularmente afectados por la pobreza, se hace necesaria por parte del Estado la prestación de servicios legales gratuitos que faciliten el acceso a instancias judiciales de protección y a una mayor difusión de información sobre recursos dentro del sistema de justicia y sobre sus derechos.

En sintesis, la $\mathrm{CIDH}$ ha identificado una serie de problemas estructurales dentro de los sistemas de justicia regionales que afectan el procesamiento de casos de violencia contra las mujeres, al igual que el juzgamiento de otras violaciones de derechos humanos, a saber:

- La ausencia de instancias de la administración de la justicia en zonas rurales, pobres y marginadas.

- Falta de abogados de oficio para las víctimas de violencia que no cuentan con recursos económicos.

- Carencia de recursos humanos y financieros para atender los problemas persistentes y estructurales.

- Debilidad institucional de los ministerios públicos y la policía que investigan los delitos con ausencia de unidades especiales dentro de las fiscalías, la policía y los tribunales con destreza técnica y conocimientos especiales.

- Precariedad y descoordinación en los sistemas de información para recopilar estadísticas sobre incidentes y casos de violencia contra las mujeres, indispensables para analizar posibles causas y tendencias y evaluar la respuesta del sistema de justicia ante actos de violencia contra las mujeres.

- Divergencia existente entre el acceso a la justicia de las mujeres basada en la raza, etnia y/o carencia o posesión de recursos económicos.

Con respecto a la carencia de recursos económicos de las víctimas se puede mencionar que entre los Estados que reportaron contar con asistencia legal gratuita se encuentran: Venezuela (a través de la Defensoría Nacional de los Derechos de la Mujer y las defensorías municipales y regionales); Perú (a través del Programa ALEgRa del Ministerio de Justicia, y el Servicio Nacional de Defensa de Oficio); Argentina (a través de los defensores de pobres, incapaces y ausentes en lo civil y comercial; de los centros de atención jurídica comunitaria dependientes de la Secretaría de Asuntos Legislativos del Ministerio de Justicia, y de los consulto-

32 oea-cidh, Equipo Latinoamericano de Justicia y Género, Centro de Estudios Legales y Sociales. "Una mirada al acceso a la justicia en los paises del Cono Sur", reunión de trabajo, presentación de Nilza Iraci, GeLEDES, Brasil, Senado de la Nación-Buenos Aires, Argentina, 12 y 13 de septiembre de 2005. 
rios jurídicos dependientes del gobierno de la ciudad de Buenos Aires y de otros organismos públicos); Brasil (las defensorías públicas ofrecen este servicio gratuito); Paraguay (a través de la Dirección SEDAmuR -Servicio de Apoyo a la Mujer- de la Secretaría de la Mujer, asiste jurídica y psicológicamente a las mujeres víctimas de violencia intrafamiliar y ofrece un servicio gratuito); República Dominicana (la Secretaría de Estado de la Mujer, a través de la Dirección de Defensoría y el Departamento de No Violencia; cada oficina provincial y municipal de la Secretaría de Estado de la Mujer trabaja en la prevención de la violencia contra las mujeres y se han puesto a disponibilidad equipos de abogadas que ofrecen de forma gratuita asesoría legal y acompañamientos a las mujeres víctimas de violencia).

Otros países, como Antigua y Barbuda, reportaron tener centros de asesoría legal para víctimas de limitados recursos, incluyendo mujeres, con un requisito de pago básico que puede ser exento en algunas circunstancias. Asimismo, en México, el Instituto Federal de la Defensoría de Oficio del Poder Judicial federal, que se rige por la Ley Federal de Defensoría Pública, ofrece un servicio gratuito e indistinto. Sin embargo, este Estado admite que no se conoce de instancias en que haya sido usado en específico por causa de discriminación o violencia en razón de género. Por último, el Estado de Belice, merced a contar con una oficina de asistencia legal que trabaja bajo la Oficina del Fiscal General, asevera que los servicios son usados, pero no en una gran medida.

Pese a lo expuesto, en la totalidad de los casos mencionados, la ciDH observa que es difícil evaluar si los programas de referencia realmente responden a las necesidades de las víctimas. Además, pudo comprobar que los ministerios públicos, la policía y los tribunales carecen de los recursos económicos y humanos indispensables para conducir investigaciones efectivas y procesar los casos hasta la etapa de sentencia, una situación particularmente crítica en las zonas rurales, marginadas y pobres.

En términos generales, en la región se advierte la ausencia de programas de capacitación y especialización para funcionarios como expertos en medicina forense, que desempeñan una labor central en el desarrollo eficaz de la prueba científica y en la presentación de la misma en el proceso penal. Pese a ello, los Estados informaron a la Comisión sobre algunos esfuerzos para proveer la capacitación necesaria. Se puede mencionar que México declara que en algunos estados, como Jalisco, Nuevo León, Chihuahua, Distrito Federal, entre otros, se han creado dentro de las unidades de medicina forense, institutos de ciencias forenses y/o procuradurías en las áreas de servicios periciales, con la especialización de médicos legistas, quienes atienden a víctimas de violencia y, en la mayoría de los casos, prestan además apoyo psicológico. 
Como se hizo referencia en el caso de la población afrodescendiente, se vuelve a poner de resalto que los vacíos y deficiencias en el procesamiento y registro de información sobre casos de violencia contra las mujeres se ven agravados porque las estadísticas oficiales en todas las esferas públicas todavía no dan cuenta de la magnitud del problema. La Comisión ha señalado su preocupación debido a que la violencia contra las mujeres, en todas sus manifestaciones y contextos, "es mucho más frecuente de lo que se cree, de lo que los medios de comunicación difunden, y de lo que las estadísticas y registros oficiales sugieren". ${ }^{33}$ Además, en numerosos países no hay una adecuada retroinformación de los tribunales al Ministerio de Justicia.

Estas carencias y debilidades en materia de información estadística sobre casos de violencia contra las mujeres se vieron reflejadas en las contestaciones de los Estados a las preguntas formuladas por la CIDH referidas a: “¿Cuántas denuncias se han recibido en los últimos 5 años sobre violaciones de derechos humanos de las mujeres, en casos de discriminación y violencia? ¿Cuántos de estos casos han llegado a la etapa de sentencia?". Varios Estados, como Brasil, Bahamas y Belice, contestaron que no disponen de esa información. Otros presentaron una serie de estadísticas en formato heterogéneo recopiladas por varias instancias estatales, como Argentina (que presentó estadísticas del Poder Judicial de la Nación, la Dirección General de la Mujer del Gobierno de la Ciudad de Buenos Aires, y la Procuración de la Corte de la Provincia de Buenos Aires); Venezuela (que presentó cifras de la Defensoría del Pueblo); Perú (que presentó estadísticas de la Sala de Familia), y México (que presentó estadísticas de la Procuraduría del Distrito Federal, las procuradurías de los estados y la Comisión Nacional de los Derechos Humanos).

Asimismo, quedó de manifiesto que la gran mayoría de los ministerios públicos y unidades especializadas de las fiscalías a nivel nacional sólo tiene cifras sobre los casos denunciados en la capital de los Estados. A modo de ejemplo, durante su visita in loco a Guatemala, la Relatoría observó que: "La invisibilización de violencia contra las mujeres era palpable en la ausencia de estudios o estadísticas sobre la prevalencia de la violencia intrafamiliar o doméstica, así como la poca información que existía sobre la magnitud de los delitos sexuales que afectan principalmente a la mujer". ${ }^{44}$ Sobre este problema, la Comisión Interamericana de Mujeres de la Organización de los Estados Americanos ha manifestado que: "la falta de datos desagregados por género y de estadísticas sobre la incidencia de la violencia, hace la elaboración de programas y el control

\footnotetext{
33 OEA-CIDH. "Las mujeres frente a la violencia y la discriminación derivadas del conflicto armado en Colombia", OEA/ Ser.L/V/I. Doc. 67, 18 de octubre de 2006, párrafo 63.

34 OEA-CIDH. "Comunicado de prensa No. 20/04", Washington, D. C., 18 de septiembre de 2004, párrafo 20.
} 
del progreso muy difícil. La falta de datos obstruye el esfuerzo por elaborar estrategias de intervención concretas". 35

Además, todo parece indicar que todavía no existe una comprensión de la relación entre las diferentes formas de violencia que pueden ser perpetradas contra una mujer: física, psicológica y sexual. Por ejemplo, en los casos de homicidios de mujeres, en muchas ocasiones no se recopila información sobre abusos sexuales que pudieron acompañar el acto perpetrado contra las víctimas. Este problema se agrava con la reticencia de las víctimas de violencia a denunciar los actos perpetrados, por el miedo a sufrir la estigmatización por parte de sus comunidades, por el tratamiento inadecuado e indiferente que pueden recibir por parte de autoridades judiciales, y por la falta de información sobre las instancias estatales a las que pueden acudir para interponer denuncias.

Ante este cuadro de situación, la ciDH destaca la necesidad de reformar la cultura judicial de una manera sostenible como una precondición para que las mujeres puedan obtener un acceso de jure y de facto a la justicia. Para ello, enfatiza la importancia de fortalecer y promover la creación de programas de capacitación homogéneos para funcionarios/as públicos, judiciales y policiales, así como también para agentes comunitarios, que presenten mecanismos de institucionalización y vigilancia necesarios para poder lograr cambios significativos, es decir, que redunden en un impacto sostenible. En este sentido, la implementación de estos programas debería acompañarse de cambios organizativos, presupuestarios, normativos, mecanismos de monitoreo y evaluación que garanticen que la puesta en marcha de los mismos "no dependa exclusivamente de voluntades personales y que conlleven niveles de institucionalización que se traduzcan en cambios sustanciales de las prácticas y en verdaderas transformaciones culturales". ${ }^{36}$ En miras a lograr tal propósito, los Estados deben adoptar medidas para sancionar a los funcionarios públicos que vulneren los derechos de las mujeres durante el proceso penal. Igualmente, la Comisión destaca la necesidad de capacitar a la población sobre el problema de la violencia contra las mujeres desde una edad temprana y formativa, para evitar la creación de estereotipos que subordinen a las mujeres.

En este contexto, resulta igualmente crítico fortalecer las políticas de prevención de los abusos y las diversas formas de violencia institucional perpetrada por autoridades estatales contra las mujeres durante el proceso judicial, como

\footnotetext{
${ }^{35}$ Comisión Interamericana de Mujeres de la OeA. "Violencia en las Américas. Análisis regional incluyendo una revisión de la implementación de la Convención Interamericana para Prevenir, Sancionar y Erradicar la Violencia contra la Mujer", octubre de 2000, p. 79.

36 oea-cidh, Centro por la Justicia y el Derecho Internacional (cejis) y Organizaciones de la Red Feminista Centroamericana. "Violencia doméstica en Centroamérica", audiencia temática, 125 Periodo Extraordinario de Sesiones, 19 de julio de 2006.
} 
un deber expreso y sin dilaciones comprendido en el artículo 7 de la Convención de Belém do Pará. Ello debido a que la mayoría de las políticas de prevención a nivel estatal se centra exclusivamente en campañas de sensibilización y difusión de información al público en general sobre los problemas de la violencia y la discriminación contra las mujeres como acciones aisladas, sin presentar un enfoque integral que abarque el sector de la justicia. Por lo tanto, las campañas de prevención deberían abordar los factores de riesgo que existen en el ámbito familiar y social, y que facilitan la aceptación de la violencia contra las mujeres por parte de los funcionarios judiciales.

lgualmente, la CIDH observa con preocupación que la mayoría de las políticas de prevención existentes continúa centrándose en la violencia doméstica, en detrimento de otras formas de violencia que ocurren en otras esferas, es decir, haciendo caso omiso a las estipulaciones de la Convención de Belém do Pará y otros instrumentos internacionales de derechos humanos. Las respuestas de los Estados ante la consulta de la CIDH sobre cuáles habían sido los mayores logros y desafíos en la implementación de las leyes y políticas públicas para prevenir, sancionar y erradicar la discriminación y la violencia contra las mujeres nos ubicaría en el seno de una cultura patriarcal reticente a los cambios. A título ilustrativo he seleccionado algunas de las contestaciones estatales: "El obstáculo más recurrente para la prevención, protección y sanción de la violencia es la persistencia de imaginarios sexistas, donde la violencia contra las mujeres tiene un alto grado de permisividad". "La aceptación cultural y silenciosa de la dominación masculina". ${ }^{38}$ "Los estereotipos, actitudes y expectativas de la sociedad hacia las mujeres continúan siendo un desafío" ${ }^{39}$.

Por lo tanto, se podría decir que los desafíos cruciales que enfrenta la Oficina de Violencia contra la Mujer son de percepción. Históricamente, el sistema de justicia criminal ha tratado la violencia contra las mujeres como un asunto privado y familiar. Sólo en las últimas dos décadas se ha reconocido el crimen de violencia contra las mujeres como un delito que requiere la fuerza y atención del sistema de justicia criminal. Otro desafío es abordar correctamente el ciclo de la violencia contra la mujer y su impacto en los hijos. Muchos Estados han comenzando a reconocer el impacto de la violencia doméstica en los niños, ya que "numerosos estudios indican que los expuestos a la misma exhiben frecuente-

\footnotetext{
${ }^{37}$ OEA-CIDH. "Respuesta del Estado de Colombia al cuestionario de la CIDH sobre la situación del acceso a la justicia de las mujeres en las Américas", 27 de enero de 2006.

38 OEA-CIDH. "Respuesta del Estado de Antigua al cuestionario de la CIDH sobre la situación de acceso a la justicia de las mujeres en las Américas", 15 de febrero de 2006.

39 OEA-CIDH. "Respuesta del Estado de Belice al cuestionario de la CIDH sobre la situación del acceso a la justicia de las mujeres en las Américas", 18 de octubre de 2006.
} 
mente mayores niveles de problemas de comportamiento, sociales y emocionales que los que no han presenciado ese tipo de violencia". ${ }^{40}$

En suma, las estrategias de prevención deben estar orientadas a reducir los factores de riesgo y aumentar los factores de protección. Ello teniendo presente que dentro de los factores de riesgo existen diversos coeficientes, a saber: a) causas estructurales como la inestabilidad laboral, la pobreza o los procesos migratorios masivos, producto de crisis económicas de regiones o países, que requieren intervenciones globales con resultados a mediano plazo; $b$ ) causas sociales como el asilamiento social o la falta de redes; c) causas familiares como las historias de violencia de cada miembro de la pareja, o d) causas individuales como la ingestión de alcohol, drogas, agresividad o valores culturales que legitimaban el uso de la violencia y situaciones de abuso de poder.

A nivel normativo, la CiDH ha verificado dos niveles de obstáculos, tanto en la legislación civil como penal, que afectan el procesamiento efectivo de casos de violencia contra las mujeres. El primero consiste en vacíos, deficiencias, falta de armonización y en la presencia de conceptos discriminatorios que colocan a las mujeres en situación de desventaja. El segundo se manifiesta a través de la falta de implementación y la incorrecta aplicación del marco jurídico existente por parte de los funcionarios judiciales.

Como se ha mencionado anteriormente, se ha verificado que en materia civil la legislación de muchos países todavía no logra abarcar las diversas manifestaciones de violencia que se cometen contra las mujeres -violencia física, psicológica y sexual-identificadas por la Convención de Belém do Pará, así como tampoco los contextos en que éstas ocurren, además del familiar (social, urbano, institucional y laboral). Las legislaciones se concentran principalmente en la violencia doméstica e intrafamiliar, colocando a las mujeres en situación de desamparo frente a otras manifestaciones de violencia. ${ }^{41}$

Asimismo, diversas legislaciones no incluyen reparaciones para las mujeres que son víctimas de violencia, a fin de que puedan ser compensadas por los daños causados. Es importante resaltar aquí que los Estados todavía adolecen de una visión homogénea de las mujeres como grupo objetivo beneficiario de políticas públicas, lo cual se traduce en un marco jurídico que no considera las necesidades particulares que requieren distintos grupos de mujeres, como las afrodescendientes y las indígenas, especialmente cuando procuran acceder a recursos judiciales con adecuadas garantías.

\footnotetext{
40 OEA-CIDH. "Respuesta del Estado de Estados Unidos al cuestionario de la CIDH sobre la situación del acceso a la justicia de las mujeres en las Américas", 31 de marzo de 2006.

${ }^{41}$ Organización Panamericana de la Salud (ops) et al. "Modelo de leyes y políticas sobre violencia intrafamiliar contra las mujeres", Washington, D. C., abril de 2006.
} 
También, la CIDH hace mención de la persistencia de disposiciones inadecuadas, y en algunos casos de contenido discriminatorio, dentro de algunas leyes y códigos civiles y penales, específicamente: definiciones de la violación, que exigen el uso de la fuerza y la violencia en lugar de la falta de consentimiento; el tratamiento de la violencia sexual contra las mujeres como un delito contra el honor y no como un crimen contra la integridad; normas procesales que establecen la terminación de los procedimientos penales cuando la víctima retira su denuncia, y sanciones insuficientes para los casos de violencia contra las mujeres. ${ }^{42}$ A modo de ejemplo, en Nicaragua, Panamá, Uruguay y Venezuela todavía existen disposiciones jurídicas que eximen al agresor de delitos sexuales si contrae matrimonio con la víctima. La CIDH ha expresado el problema del siguiente modo:

En numerosos códigos penales, valores tales como la honra, el pudor social, la doncellez, la castidad, las buenas costumbres, prevalecen sobre valores como la integridad psicofísica y la libertad sexual, impidiendo así la debida protección legal a las víctimas de tales delitos, u obligándolas a probar que opusieron resistencia en el caso del delito de violación, o sometiéndolas a procedimientos interminables que producen una continua victimización. ${ }^{43}$

Sumado a ello, existen casos en donde dentro de un mismo marco jurídico nacional se ha identificado la falta de armonización entre disposiciones contenidas en distintas leyes especiales, lo cual genera desconcierto. Por ejemplo, en Paraguay, "existe contradicción entre las disposiciones del Código Penal y las incluidas en la Ley 1600 sobre violencia doméstica”. ${ }^{44}$ Mientras que la Ley 1600 ofrece varias alternativas para presentar una denuncia sobre un acto de violencia, ya sea ante la policía, centros de salud o juzgados de paz, la legislación penal establece que el ingreso de las denuncias se realiza por la intervención de oficio del Ministerio Público, o mediante denuncias ante el mismo o la policía. La multiplicidad de alternativas crea confusión en las víctimas, sobre todo las que no tienen experiencia previa o conocimiento de los procedimientos judiciales.

Por otro lado, la CIDH ha tomado conocimiento de la resistencia y del desconocimiento de algunos jueces sobre la aplicación e interpretación de tratados internacionales de derechos humanos en el derecho interno. Las irregularidades son descritas de la siguiente forma:

42 NACIONES UnIDAS. Informe del secretario general: "Estudio a fondo sobre todas las formas de violencia contra la mujer". [En línea]. A/61/122/Add.1, 6 de julio de 2006, párrafo 277. [Citado: 30. Enero. 2007]. Disponible en: http:// www.un.org/womenwatch/daw/.

43 OEA-CIDH. "Informe de la Comisión Interamericana sobre la condición de la mujer en las Américas", OEA/SER.L/V/I.98, Doc. 17, 13 de octubre de 1998, sección IV, conclusiones.

44 Idem. 
La falta de compromiso de los gobiernos; las situaciones de inestabilidad política; la predominancia, en el nivel nacional, de una cultura jurídica poco favorable al derecho internacional de los derechos humanos, en especial en las cuestiones relacionadas con la discriminación basada en el género; el desconocimiento del significado, contenido e importancia de tales tratados por parte de los(as) parlamentarios(as) que los aprueban; la resistencia a cambios de fondo, a inversiones presupuestarias en esa área y a la aceptación de mecanismos de accountability; la debilidad de los mecanismos institucionales; la gran fuerza de sectores religiosos conservadores y fundamentalistas, como algunos de la lglesia católica; la insuficiente movilización y presión de sectores organizados de la sociedad. ${ }^{45}$

Por ello, es vital efectuar una evaluación y un seguimiento periódico de la legislación de los Estados para asegurar que los servidores públicos la apliquen en forma correcta, particularmente los miembros del ministerio público y del Poder Judicial. Sería deseable que fuera común la utilización de mecanismos de evaluación de los procesos de implementación de leyes de prevención de violencia contra las mujeres y la fiscalización externa de la actuación de los ministerios públicos y de los funcionarios judiciales en términos de indicadores de eficacia y de resultado.

Por último, la CIDH observa que es imposible que las mujeres puedan reivindicar sus derechos desde la ignorancia. En particular, se ha constatado que las mujeres de escasos recursos desconocen sus derechos y los mecanismos existentes para hacerlos valer. La falta de información sobre los recursos judiciales disponibles y el hecho de que la violencia y la discriminación contra las mujeres todavía sean conductas aceptadas en las sociedades americanas, dan como resultado un reducido número de denuncias de actos de violencia de género. Como modo de solución, enfatiza la necesidad de desarrollar programas educativos para el público en general sobre los derechos humanos y sobre los recursos judiciales existentes para la interposición de denuncias.

Ante este cuadro de situación, la CIDH recomienda la implementación de las siguientes medidas como elemento clave para prevenir, sancionar y erradicar la discriminación y la violencia contra la mujer:

1) Erradicar definitivamente conceptos y valoraciones discriminatorios en las leyes; por ejemplo, en los delitos sexuales, conceptos tales como castidad y honestidad, o la posibilidad de que cese la acción penal si el victimario contrae matrimonio con la víctima.

\footnotetext{
45 Pandjarjan, Valeria e Hirao, Denise. "Balance sobre la situación de la violencia doméstica en la subregión Brasil y Cono Sur, Argentina, Brasil, Chile, Paraguay y Uruguay". [En línea], São Paulo, Brasil, junio de 2004. [Citado: 3. Mayo. 2014]. Disponible en: http://www.cladem.com/espanol/regionales/Violenciadegenero/Proyecto/balanconosur.asp.
} 
2) Formar a los operadores del sistema de justicia (agentes del Ministerio Público, policías, médicos legistas, servidores públicos y peritos) en el respeto de los derechos humanos de las mujeres y la observancia de la perspectiva de género.

3) Incluir la perspectiva de género en los planes curriculares desde la formación inicial de los niños y las niñas, así como fomentar la creación de políticas públicas libres de estereotipos de género y de sexismos. La permanencia y la legitimación de la violencia familiar se debe en gran medida a las prácticas culturales subyacentes de las relaciones de género, mismas que comúnmente se basan en "mitos, estereotipos de inferioridad, dominio, abnegación y control de las mujeres frente a los hombres”.

\section{Reflexión final}

Merced las crudas y lamentables realidades expuestas con anterioridad en perjuicio del derecho humano universal a la igualdad de género, estimo que la difícil situación de las mujeres en las Américas ha mejorado en los últimos años debido a que la violencia que padecen, junto a la multifacética discriminación que soportan, ha pasado de la más absoluta invisibilidad a la visualización, la aceptación del reclamo y, en sendos casos, la reparación de los agravios. Particularmente, considero que el papel que ha jugado la CIDH ha sido fundamental para la transversalización de la perspectiva de género en todas las aéreas. Pese a ello, es deseable que este esfuerzo sea incrementado día a día mediante un trabajo mancomunado de la sociedad civil (familia, escuelas, organizaciones no gubernamentales, etcétera), los tres poderes de cada Estado, los organismos internacionales y regionales y la comunidad internacional, que bregue por la implementación y aplicación del plexo normativo nacional y supranacional tendente al respeto irrestricto de los estándares internacionales que garanticen en la región una legítima y consolidada igualdad de género. 